\title{
Intestinal absorption of $\beta$-glucans and their effect on the immune system
}

\author{
Shingo Hino ${ }^{1 *}$, Naomichi Nishimura ${ }^{1}$, Tsukasa Matsuda ${ }^{2}$ and Tatsuya Morita ${ }^{1}$ \\ 1 College of Agriculture, Academic Institute, Shizuoka University, Shizuoka 422-8529, Japan \\ 2 Graduate School of Bioagricultural Sciences, Nagoya University, Nagoya 464-8601, Japan; Faculty of Food \\ and Agricultural Sciences, Fukushima University, Fukushima 960-1296, Japan \\ * Correspondence: hino.shingo@shizuoka.ac.jp; Tel.: +81-54-238-4820
}

\begin{abstract}
: $\beta$-glucan is a generic term for insoluble dietary fibers exerting various effects on the immune system. As a group, $\beta$-glucans are non-cellulose polysaccharides composed of a glucopyranose as the main constituent sugar with $\beta$ configuration, having a $\beta$ - $(1,3)$-linked glucopyranose main chain as a common feature. $\beta$-glucans are absorbed through the intestine. Since the 1980s, there have been many studies reporting various effects of $\beta$-glucans on the immune system, including reports on receptors, that have slowly clarified their recognition system and action mechanisms. However, these studies focused mostly on treatments of infectious diseases and tumors; thus, the effects of $\beta$-glucans ingested in food as dietary fiber and their mechanisms of action remain largely unknown. The uptake of $\beta$-glucan into the body may be resemble that of proteins, which are soluble polymers, and insoluble material such as dietary fiber. Dietary fibers have varied structures, with wide-ranging solubility and physiological effects. Understanding whether these substances are actually taken up, how they exert their effects, and their metabolism after being taken up are important issues when considering the functionality and safety of dietary fibers.
\end{abstract}

Keywords: $\beta$-glucan; intestinal absorption; immune system

\section{What is a $\beta$-glucan?}

$\beta$-glucan is a generic term for non-cellulose polysaccharides composed of a glucopyranose as the main constituent sugar with $\beta$ configuration, and it has a $\beta$ - $(1,3)$-linked glucopyranose main chain as a common feature. Although many $\beta$-glucans have $\beta-(1,6)$-linked glucopyranose branched chains attached to the main chain, the frequency of branched chains varies by species (Table 1) [1-8]. For instance, Schizophyllan from Schizophyllum commune has a $\beta-(1,3)$-linked main chain and one $\beta-(1,6)$ linked glucopyranose side chain at every third glucose unit [4]. In contrast, Grifolan from Grifola frondosa has two $\beta$-(1,6)-linked glucopyranose side chains at every five glucose units [2]. There are also linear chain $\beta$-glucans without side chains such as Curdlan from microorganisms and Callose in plant cell walls, and these are known as $\beta-(1,3)$ glucans without branched chains [1]. Another structure observed in barley $\beta$-glucans such as the linear chain $\beta-(1,3)(1,4)$ glucan is a structure where $\beta-(1,3)$ linkages and $\beta-(1,4)$ linkages are irregularly arranged in longitudinal rows. Broadly speaking, $\beta$-glucans include $\beta$-glucan-containing complexes that are not composed of single-type molecules such as yeast-derived Zymosan , a complex consisting of $\beta-(1,3)(1,6)$ glucan, mannan, and proteins [6], and Trametes versicolor-derived Krestin , a $\beta$ - $(1,3)$ glucan complex with proteins [7].

$\beta$-glucans are water insoluble, except for those with low molecular weight or those that form complexes with proteins, and they are known to dissolve in alkaline solutions and solvents such as dimethyl sulfoxide. Although $\beta$-glucans generally have a stable triple helical structure, a nuclear magnetic resonance (NMR) analysis reported that the structure changes to a single helix in an alkaline 
solution. The details of the relationship between the three-dimensional helical structure of the $\beta$ glucan main chain and biological activity remain unknown.

Table 1. Types and structures of $\beta$-glucans

\begin{tabular}{|c|c|c|c|}
\hline & Source & Structure & Reference \\
\hline Curdlan & Alcaligenes faecalis & Linear $\beta-(1,3)$ glvcan & McIntosh, Stone \& Stanisich, 2005 [1] \\
\hline Grifolan & Grifola frondosa & $\begin{array}{l}\text { Linear } \beta-(1,3) \text { glvcan with } \beta-(1,6) \text { glvcan branching } \\
\text { for every five }(1,3) \text { Glcp residues }\end{array}$ & Munz, Steinman \& Fujii, 2005 [2] \\
\hline Lentinan & Lentinula edodes & $\begin{array}{l}\text { Linear } \beta-(1,3) \text { glvcan with } \beta-(1,6) \text { glvcan branching } \\
\text { for every three }(1,3) \text { Glcp residves }\end{array}$ & Sasaki \& Takasuka, 1976 [3] \\
\hline Schizophyllan & Schizophylleoncomme & $\begin{array}{l}\text { Linear } \beta-(1,3) \text { glvcan with } \beta-(1,6) \text { glvcan branching } \\
\text { for every three }(1,3) \text { Glcp residves }\end{array}$ & Tabata K et al., 1981 [4] \\
\hline Scleroglvcan & Sclerotinia sclerotionton & $\begin{array}{l}\text { Linear } \beta-(1,3) \text { glvcan with } \beta-(1,6) \text { glvcan branching } \\
\text { for every three }(1,3) \text { Glcp residves }\end{array}$ & Garcia-Lora et al.,2003 [5] \\
\hline Zymosan & Saccharompees cerevisiae & Complex of $\beta-(1,3)(1,6)$ glvcan and mannan & Sato et al.,2003 [6] \\
\hline Krestin & Trametes versicolar & Complex of $\beta-(1,3)(1,6)$ glvcan and proteins & Kurashige, Akuzawa \& Endo, 1997 [7] \\
\hline Epiglucan & Epicoccumnigrum & $\begin{array}{l}\text { Linear } \beta-(1,3) \text { glvcan with two } \beta-(1,6) \text { glvcans } \\
\text { branching for every three }(1,3) \text {-Glcp residues }\end{array}$ & Schmid F et al.,2001 [8] \\
\hline
\end{tabular}

\section{The effects of $\beta$-glucans on the immune system}

The physiological effects of microbial components have long been known through medicinal fungi such as cordyceps and reishi mushrooms. Some of the components of these medicinal fungi are conserved in lower species such as microorganisms; however, since they are not found in animals, living organisms recognize them as Pathogen Associated Molecular Patterns (PAMPs) and activate both innate and acquired immunity. $\beta$-glucans are structures conserved as cell wall components of fungi and yeasts, and they are recognized by the immune system cells and act as PAMPs. Research on $\beta$-glucans began in the 1940s when a component with complement-activating capacity called Zymosan was identified in the extract of the cell wall of baker's yeast. Research in the 1960s and 1970s identified that $\beta-(1,3)(1,6)$ glucan molecules were the components in Zymosan stimulating the immune system. Today, using the effect on the immune system, $\beta$-glucans such as Lentinan, a mushroom-derived $\beta$-glucan, Schizophyllan, and Krestin have been approved and used as supplemental agents with antitumor medication.

\subsection{Effects on innate immunity}

Innate immunity is the immune system that we are born with, and it is mainly responsible for the early immune response against infections and tumors. A study suggested that $\beta$-glucans activate phagocytes such as macrophages and dendritic cells to increase their ability to kill phagocytosed microbes following infections and remove dead cells [9]. Studies have shown that $\beta$-glucans activate natural killer (NK) cells to effectively remove tumor cells and virus-infected cells [5], act on neutrophils, and enhance the bactericidal activity against Streptococcus pneumoniae [10]. Whereas many of these studies reported the results of administering $\beta$-glucan into the blood or in cultured cell systems, there are also studies reporting the efficacy of oral administration in experimental animals. Suzuki et al. observed the activation of $\mathrm{T}$ cells, NK cells, and macrophages with 10-day oral administration of $\beta$-glucan in mice, and they reported the suppression of growth of the implanted tumor cells [11]. Hong et al. demonstrated that oral administration of $\beta$-glucans to mice induced neutrophil activation and enhanced the inhibitory effect of tumor cell growth by tumor antigenspecific monoclonal antibodies [12]. 


\subsection{Effects on acquired immunity}

Whereas $\beta$-glucans activate various cells and induce innate immune responses, they are also known to act on acquired immunity. Acquired immunity is mediated primarily by B cells and T cells. $B$ cells are mainly involved in the antibody response (humoral immune response), and $\mathrm{T}$ cells are responsible for the cellular immune response. Acquired immunity requires antigen presentation by phagocytes such as dendritic cells and macrophages. In addition, various cytokines that are secreted by immune cells, mainly $\mathrm{T}$ cells, are essential for the differentiation and maturation of $\mathrm{T}$ cells upon antigen presentation and for the immunoglobulin (Ig) class switching of B cells. $\beta$-Glucans are known to induce the production of various cytokines through the activation of dendritic cells and macrophages, and the produced cytokines are involved in the differentiation of helper $\mathrm{T}$ cells (Th cells). Cytokines produced by $\beta$-glucan-stimulated dendritic cells in mice induced the differentiation of Th cells, primarily Th1 and Th17 [13]. A study reported that oral administration of yeast-derived Zymosan and antigens in mice induced antigen-specific regulatory $\mathrm{T}$ cells $\left(\mathrm{T}_{\mathrm{reg}}\right)$ and established antigen-specific oral immune tolerance [14]. This suggested that the induction mechanism and effect of $\mathrm{T}$ cells may differ between oral and non-oral routes.

In a human clinical study, oral administration of Zymosan increased the production of immunoglobulin A (IgA) secreted into the intestinal tract and saliva [15]. The fact that Krestin is used as an oral medication suggests that oral administration of $\beta$-glucans also has an effect on the immune systems of humans. However, it is not clear whether the activation of the immune system by these given orally occurs after $\beta$-glucan is absorbed into the body and is triggered by immune cells present in sites such as the lamina propria, or whether $\beta$-glucans indirectly induce the activation of immune system cells when the unabsorbed $\beta$-glucan activates the intestinal epithelial cells.

\subsection{Antibody response and recognition specificity for $\beta$-glucan}

Although antibodies play a major role in acquired immunity, $\beta$-glucans act to modify the antibody response towards common protein antigens and themselves become antigens. For instance, the levels of immunoglobulin $\mathrm{M}(\operatorname{IgM})$ and immunoglobulin $\mathrm{G}(\operatorname{IgG})$ in the blood increased over time when DBA/2 mice were immunized with Curdlan $(\beta-(1,3)$ glucan), Zymosan $(\beta-(1,3)(1,6)$ glucan), and Pustulan $(\beta-(1,6)$ glucan) (Figure 1A) without an adjuvant. In general, polysaccharide antigens are Tcell-independent antigens and can differentiate into plasma cells (antibody-producing cells) by directly activating B cells. However, they do not activate T cells, and antibody class switching is not induced; as a result, IgM predominates. As mentioned previously, however, $\beta$-glucans induce an IgG response. Since $\beta$-glucans induce the production of various cytokines through their action on immune system cells, $\beta$-glucans themselves act as adjuvants inducing antibody responses, and it is further presumed that they may trigger antibody class switching. Other than being recognized as an antigen, this action of $\beta$-glucans as antigens differs from common protein antigens that do not stimulate the immune system themselves. The antibodies produced in this matter also identify specific glycosidic linkage patterns for each $\beta$-glucan (Figure 1B).

Antibodies that recognize $\beta$-glucans were also found in the serum of healthy subjects [16]. This suggested that an antibody-mediated mechanism may exist to recognize and remove $\beta$-glucans from 
the body. It is also possible that an immune complex of $\beta$-glucans and specific antibodies acts on the immune system.

(a)

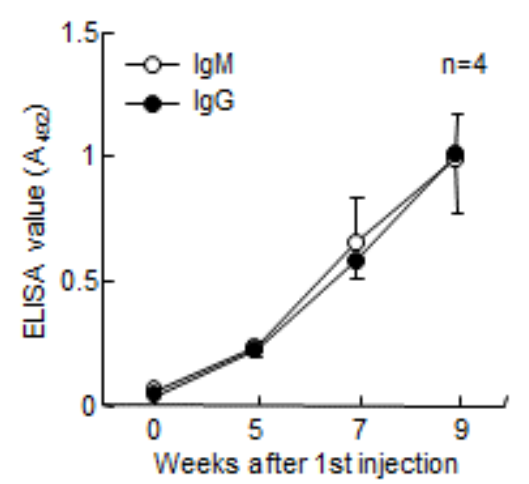

(b)

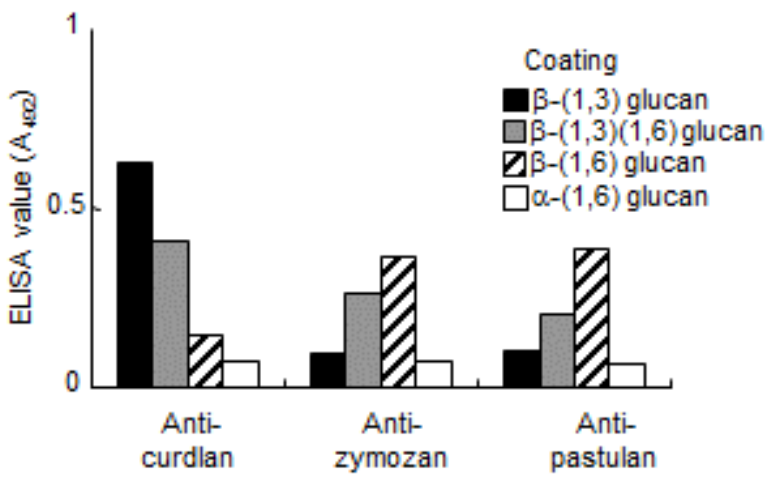

Figure 1. Antibody responses and reactive specificities in DBA/2 mice immunized with $\beta$-glucans. (A) DBA/2 mice were intraperitoneally administered $1 \mathrm{mg}$ of curdlan every 2 weeks without adjuvant. Changes in anti- $\beta$-(1,3)-glucan IgM and IgG titer over time were observed by ELISA. (B) DBA/2 mice were immunized with curdlan $(\beta-(1,3)$ glucan), zymosan ( $\beta$ $(1,3)(1,6)$ glucan) and pastulan $(\beta-(1,6)$ glucan), respectively. The reactive specificity of each IgG antibody was analyzed by ELISA.

\section{3. $\beta$-glucan receptors}

In recent years, progress has been made in research on molecules involved in innate immunity called Pattern Recognition Receptors (PRRs), including Toll-Like Receptor (TLR). TLR2, complement receptor 3 (CR3), and dendritic-cell-associated C-type lectin-1 (Dectin-1) have been identified as candidate receptors that recognize $\beta$-glucans (Table 2). TLR2 is a TLR family protein that extracellularly has a leucine-rich repeat domain that is common to the TLR family. Mediated by this extracellular domain, TLR2 has been identified as a receptor that recognizes lipoteichoic acids and peptidoglycan, cell wall components of Gram-positive bacteria. Subsequently, it was reported that TLR2 also binds to Zymosan, a $\beta$-glucan, to induce cytokine production [6]. However, it was shown that there was no binding with $\beta-(1,3)(1,6)$ glucan purified from Zymosan, and it is now believed that

Table 2. Summary of $\beta$-glucan receptors

\begin{tabular}{|c|c|c|c|}
\hline Receptor & Cell types & Major functions & Ligands other than $\beta$-glvcan \\
\hline Dectin-1 & $\begin{array}{l}\text { Mononvclear cell, } \\
\text { macrophage, neutrophil, } \\
\text { dendritic cell, T cell }\end{array}$ & $\begin{array}{l}\text { Phagocytosis, ROS and cytokine } \\
\text { production }\end{array}$ & $\begin{array}{l}\text { Fungi, } T \text { cell membrane } \\
\text { protein (unidentified) }\end{array}$ \\
\hline $\begin{array}{l}\text { Complement receptor } 3 \\
\text { (CD11b/CD18) }\end{array}$ & $\begin{array}{l}\text { NK cell, neutrophil, } \\
\text { B cell, T-cell }\end{array}$ & Cancer cytotoxicity & Various microbiota \\
\hline Scavenger receptor & $\begin{array}{l}\text { Endothelial cell, } \\
\text { Myeloid cells }\end{array}$ & Phagocytosis & LDL, HDL \\
\hline Lactosylceramides & $\begin{array}{l}\text { Neutrophil, } \\
\text { epithelial cells }\end{array}$ & ROS and cytokine production & Various microbiota \\
\hline Toll-likereceptor 2 & $\begin{array}{l}\text { Macrophage, dendritic } \\
\text { cell, B cell, } T \text { cell, } \\
\text { epithelial cells }\end{array}$ & Cytokine production & Various microbiota \\
\hline
\end{tabular}

HDL, high density lipoprotein; LDL, low density lipoprotein; ROS, reactive oxgen species.

TLR2 is not a receptor that recognizes single $\beta$-glucans [17].

CR3, a complement receptor expressed primarily by neutrophils, is a heterodimeric protein consisting of CD11b and CD18. It has a lectin-like domain in the extracellular domain, separate from 
the complement-binding site, and uses this domain to bind to $\beta$-glucans [18]. CR3 binding to $\beta$ glucans is reported to increase signaling into the cell by complement binding, increasing the cytotoxic activity against opsonized pathogenic microorganisms and tumor cells [19].

Dectin-1, known to play a major role when macrophages and dendritic cells recognize $\beta$-glucans, is a type II single transmembrane protein that has an NK cell receptor-like C-type lectin-like domain in the extracellular domain. It uses this part to recognize and bind to $\beta$-glucans. Dectin- 1 also has an immunoreceptor tyrosine-based activation motif (ITAM) in the intracellular domain that undergoes tyrosine phosphorylation by binding to $\beta$-glucans and activates cells through various signaling pathways [20].

Although there are many different types of receptors for $\beta$-glucans, multiple types of $\beta$-glucan receptors seem to act collaboratively in their response to $\beta$-glucans. In fact, in responding to Zymosan, TLR2 and Dectin-1 act collaboratively and strengthen the immune response [21].

This section introduces recent studies conducted by the authors' group on Dectin-1, a major $\beta$ glucan receptor, and binding specificity with $\beta$-glucans.

\subsection{Specificity in recognizing glycosidic linkage patterns of $\beta$-glucan receptor Dectin-1}

An extracellular domain with a $\beta$-glucan binding site of mice, Dectin-1, was prepared using a fruit fly-derived S2 cell to analyze binding of various glucans to this soluble receptor (Dectin-1CRD-V5His) (Figure 2).

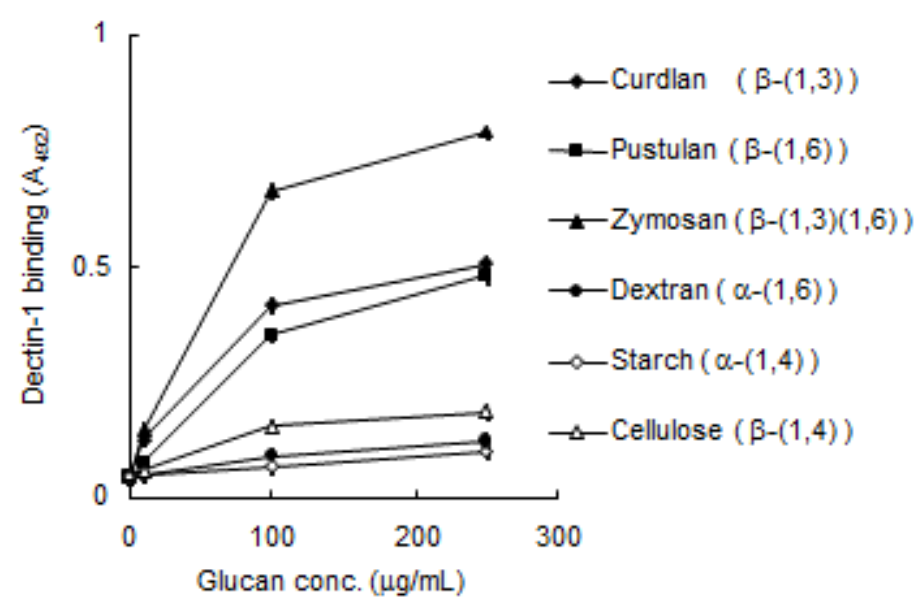

Figure 2. Specificity of the recombinant mouse Dectin-1. The binding of the recombinant mouse Dectin-1 (Dectin-1 CRD-V5-His) to various glucans was analyzed. $0-250 \mu \mathrm{g} / \mathrm{mL}$ of various glucans were coated and the binding of the recombinant Dectin-1 was analyzed.

Dectin-1CRD-V5-His expressed in S2 cells specifically bonded to $\beta$-glucans with $\beta$ - $(1,3)$ or $\beta-(1,6)$ linkages. Zymosan, which is a $\beta-(1,3)(1,6)$ glucan, had a higher binding ability with Dectin-1CRD-V5His compared to Curdlan, a $\beta-(1,3)$ glucan, and Pustulan, a $\beta-(1,6)$ glucan, suggesting that glucans with $\beta-(1,6)$ branched chains form stronger linkages with Dectin-1. These results were similar to the results obtained by Gordon et al. [22], who investigated the inhibiting effects of various $\beta$-glucans in the binding of Dectin-1 and Zymosan, and the affinity of Dectin-1 seems to be higher in the order of $\beta$ - $(1,3)(1,6)$ glucan, $\beta$ - $(1,3)$ glucan, and $\beta$ - $(1,6)$ glucan. Previous studies have suggested that $\beta$-glucans 
with $\beta-(1,6)$ branched chains have high immune activation, and one possible explanation for this may be the affinity with Dectin-1.

\subsection{The association between Dectin-1 binding and $\beta$-glucan chain length}

Many $\beta$-glucans are cell wall components, and they vary in size depending on their origin and extraction process. Curdlan was fractionated into large and small particle size $\beta$-glucans by centrifugation to compare macrophage activating ability, and the results of comparing the same added amounts showed that smaller $\beta$-glucan particles had higher macrophage activating ability. Even small $\beta$-glucan particles seem to be aggregates of $\beta$-glucans of various chain lengths, and of these molecules with different chain lengths, it is not known which chain lengths of $\beta$-glucans have strong bioactivity. Natural $\beta$-glucans have long chains, and they form aggregates that are waterinsoluble particles. Thus, it is difficult to prepare and isolate $\beta$-glucans with varying chain lengths as single molecular species.

Subsequently, we prepared a soluble, low molecular $\beta$-glucan by acid hydrolysis of granular $\beta$ $(1,3)$ glucan (Curdlan) with $100 \mathrm{mM}$ hydrochloric acid at $100{ }^{\circ} \mathrm{C}$, and $\beta$-glucans with different chain lengths were fractionated by molecular sieve chromatography. Measurement of hexoses by the phenol sulfate method detected three peaks. Fractions eluted in the first exclusion volume peak (A) and the second peak (B) had a spectroscopic property characteristic of $\beta$-glucans that emit fluorescence at around $500 \mathrm{~nm}$ when interacted with aniline blue. In contrast, no fluorescence was detected in the fraction of the third peak even when aniline blue was added. When these fractions were analyzed with matrix-assisted laser desorption ionization-time of flight-mass spectrometry (MALDI-TOF-MS), we found that, due to hydrolysis, fractions nos. 13 to 20 contained fractionated low molecular $\beta$-glucans with chain lengths of three to 15 glucose units.

When binding to Dectin-1 was analyzed using the obtained fractions, we found that all fractions bound to Dectin-1 in a concentration-dependent manner, and the affinity was higher with longer chains. However, binding was also confirmed in fraction No. 20, which had a $\beta$-glucan with a chain length of three to four glucose units as a main component (Figure 3).

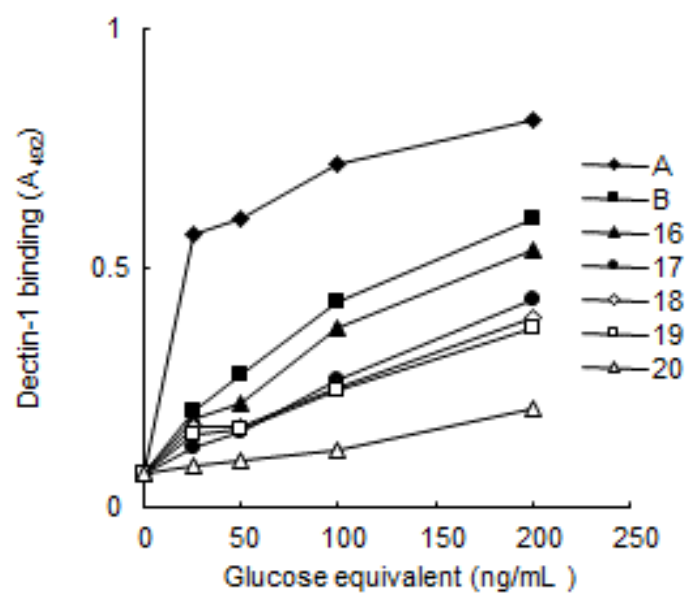

Figure 3 Binding of recombinant mouse Dectin-1 to lowmolecular-weight soluble $\beta$-glucans. Analysis of the binding of each fraction of low-molecular-weight soluble to recombinant mouse Dectin-1 was performed by sandwich ELISA. 96-well plates were coated with Dectin-1 CRD-V5-His and then each fraction was added at 0-250 ng/mL. Detection was performed using Fc-Dectin-1, PODanti-human IgG.

The activation of nuclear factor kappa B (NFkB) (Figure 4) in macrophages and the ability to induce cytokine expression were used as indicators to determine whether these soluble, low molecular weight $\beta$-glucans with Dectin-1 binding actually have the ability to activate immune cells. The activating potential of $\mathrm{NF} \kappa \mathrm{B}$ was strong in proportion to the chain length of $\beta$-glucans when 
measured under the same weight concentration conditions. As for specific activity per mole, which took into consideration the molecular weight of the different chain lengths, chain length seemed to have an even greater impact than the results shown in Figure 4.

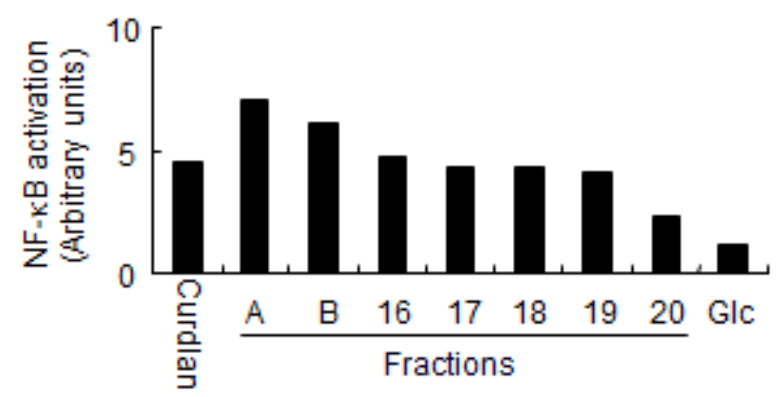

Figure 4 Macrophage activation capacity of low molecularly soluble $\beta$-glucans. RAW264.7 cells stably transfected with the NF-kB reporter gene were stimulated with low-molecular-weight $\beta$-glucans, curdlan, and glucose, respectively, at a concentration of $25 \mu \mathrm{g} / \mathrm{mL}$ for 16 hours. The ability to activate NFkB was measured by measuring fire fly luciferase activity.

Fractions $\mathrm{A}$ and $\mathrm{B}$ also tended to have higher activity per unit weight than the granular, insoluble $\beta-(1,3)$ glucan.The reason why fractions $A$ and $B$ had stronger activating potential of $N F \kappa B$ compared to insoluble $\beta$-glucans was not necessarily due to the difference in physical properties of solubility or insolubility in water, but it was most likely because fractions A and B were more highly diffused in the solution and were able to activate more cells. Even when the ability to induce cytokine expression was used as an indicator, fractions A and B showed strong activity, and moreover, there was even activation potential in fractions with three to four sugar structures. However, a clear difference in inductive capacity of sugar chain length was not detected with the semi-quantitative reverse transcription polymerase chain reaction (RT-PCR). These results suggested that mice Dectin-1 can recognize low molecular $\beta$-glucans of about three to four glucose units and has the ability to act on the immune system.

Although the amino acid sequence identity of the entire sugar chain recognition site for mice Dectin-1 and human Dectin-1 is about $60 \%$, the location of disulfide bonds and the amino acid sequences that directly interact with $\beta$-glucans seem to have been conserved between species. According to Ujita et al.'s group, soluble human Dectin-1 can also recognize $\beta$-glucans with chain lengths of three of more glucose units, similar to mouse Dectin-1 [23]. Although the overall amino acid sequence of Dectin-1's sugar chain recognition site is not very similar, there is probably not a large difference in the specificity of $\beta$-glucan recognition between species.

\section{Intestinal absorption of $\beta$-glucans}

Though $\beta$-glucans are pathogen-derived non-self molecules (foreign substances) in infection and defense responses, they are also components found in food as dietary fiber. The fact that minuscule amounts of soluble proteins are absorbed through the digestive tract and delivered to the periphery through the bloodstream is a physiological phenomenon that has long been known, and it has been reported that the amount is sufficient to trigger an immune response [24]. However, there are surprisingly few studies that have examined the dynamics of polysaccharides (dietary fiber) such as 
$\beta$-glucans in the digestive tract and their absorption from the intestinal tract into the body. Many of the studies investigating the effect of oral $\beta$-glucan administration on the body have used antitumor activity or resistance to infection as indicators of physiological effects and have not focused on intestinal absorption or action mechanisms. There has been a study that focused on intestinal absorption, and it is an example of a quantitative analysis. Rice et al administered a fluorescently labeled soluble $\beta$-glucan intragastrically to mature rats with a sonde and quantitatively measured fluorescence in peripheral blood [25]. This study used three types of soluble $\beta$-glucans with different degrees of polymerization and functional group modification and found that the time for the peripheral blood concentration to peak varied with different molecular weights and structures. However, the fact that this study measured fluorescence and did not actually measure $\beta$-glucan itself needs to be taken into consideration. In addition, the mechanism of where and how orally ingested $\beta$-glucans are taken up into the body and reach the peripheral blood is yet to be identified.

In the small intestine, the uptake of materials from the intestinal lumen is carried out by villi, which mainly absorb nutrients, as well as by the Peyer's patches, which play a central role in the intestinal immune system. The microfold cells (M cells) of the Peyer's patches within the small intestine actively take up high-molecular weight components in the intestinal lumen and contribute to the homeostasis of the intestinal immune system by actively supplying the microbes themselves or the microbial components to immune-related cells. Now, let us focus on the villi. It has been suggested that the intraluminal materials on the apical side of the intestinal epithelial cells of the villi are taken up intracellularly by endocytosis, and through intracellular vesicle transport, they are released by exocytosis from the basolateral side into the lamina propria using active transport systems such as "transcytosis" or passively transported by "paracellular transport" in which materials are taken up between the gaps of loosened tight junctions.

This section presents the authors' recent studies where soluble Dectin-1 was used as a specific probe for $\beta$-glucans to study the absorption pathway and transport mechanism of $\beta$-glucans in mice and human intestinal epithelial cell lines.

\subsection{Experiments with mice}

For seven days, Curdlan, a $\beta-(1,3)$ glucan, was intragastrically administered to mice using a sonde, and the abdomen was opened 24 hours after the last dose. The distribution of $\beta$-glucans in the tissue of the small intestine was analyzed by an immunohistological approach that used soluble Dectin-1 as a probe. $\beta$-glucans were predominantly observed in the Peyer's patches of the small intestine (Figure 5A, B), suggesting that $\beta$-glucans are taken up into the body through $\mathrm{M}$ cells. Costaining the internalized $\beta$-glucans and macrophage markers showed that most of the $\beta$-glucans in the Peyer's patches had been taken up by macrophages. In addition, these macrophages had expressed the $\beta$-glucan receptor Dectin-1, suggesting that it was taken up into the cell by phagocytosis through this receptor. In some villi of the small intestine the accumulation of $\beta$-glucan was observed in an area thought to be lymphatic vessels, and thus it seems that $\beta$-glucans taken up by the small intestinal villi enter the bloodstream through lymphatic vessels to reach peripheral tissues.

Since $\beta$-glucan uptake was observed in the intestinal tissue, $\beta$-glucan concentration in peripheral blood 24 hours after the last dose was measured. There was a significant increase in blood $\beta$-glucan 
concentration in the treated group compared to the non-treated group. These results showed that some $\beta$-glucans administered orally are absorbed through the intestinal tract and reach the peripheral blood. To investigate whether $\beta$-glucans are transported in the small intestinal epithelial cells and to analyze their transport pathway, Curdlan was administered into the duodenal lumen of mice. Three hours later, we analyzed the $\beta$-glucan distribution in the small intestinal villi with immunostaining and confirmed $\beta$-glucan uptake into the small intestinal epithelial cells in the villi (Figure 5 C, D). These results suggested that intestinal epithelial cells take up $\beta$-glucan into the body through transcellular transport. Furthermore, the $\beta$-glucans observed in the small intestinal epithelial cells were smaller than the $\beta$-glucans in the Peyer's patches phagocytosed by macrophages. Generally, M cells in the Peyer's patches are known to be able to engulf larger particles compared to the small intestinal epithelial cells, suggesting that there may be different absorption pathways depending on particle size.

(a)

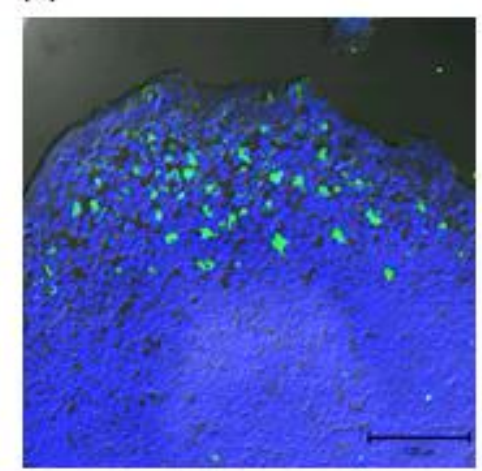

(c)

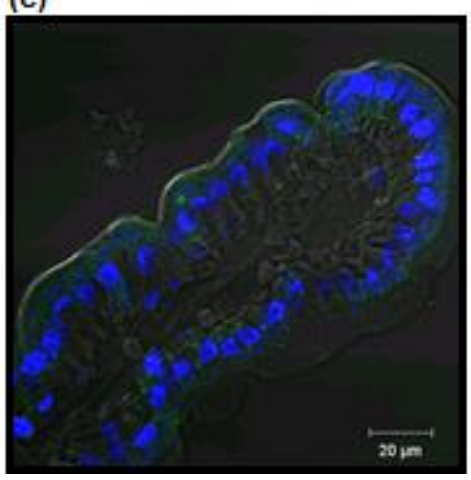

(b)

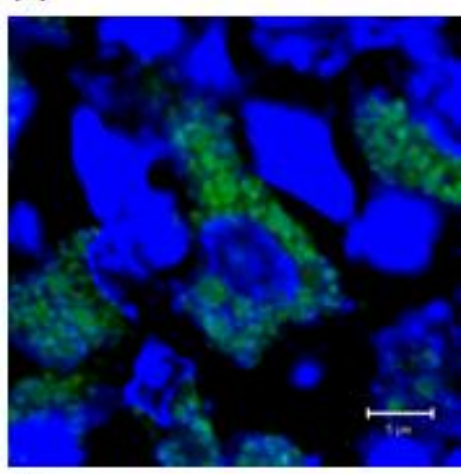

(d)

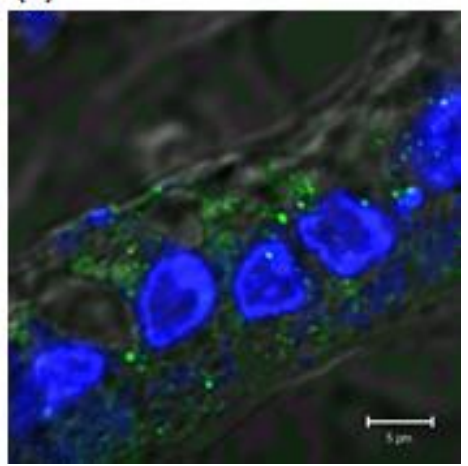

Figure 5 Intestinal absorption of $\beta$-glucan in DBA/2 mice. $\beta$-Glucan was stained with Dectin-1 CRD-V5-His (green) and nuclei were stained with TOTO-3 (blue). Observations were performed using a confocal microscope. (A, B) Curdlan $1 \mathrm{mg} /$ day was intragastorically administered to DBA/2 mice for 1 week. Twenty-four hours after the last dose, the small intestine was removed and frozen sections $(10 \mu \mathrm{m})$ of the Peyer's patch were prepared. (C,D) Curdlan $1 \mathrm{mg}$ was administered into the duodenum of DBA/2 mice. Three hours after administration, the small intestine was removed and frozen sections $(10 \mu \mathrm{m})$ of epithelial tissue were prepared.

\subsection{Experiments using the human intestinal epithelial-like cell line Caco-2}

Since $\beta$-glucan administered to the small intestinal tissue of mice was taken up in the small intestinal epithelial cells, we subsequently analyzed the absorption of $\beta$-glucans by intestinal epithelial cells in an in vitro intestinal epithelial model using human intestinal epithelial cell line Caco2 cells. After a Caco-2 cell monolayer $\left(4.67 \mathrm{~cm}^{2}\right)$ was cultured on a semi-permeable membrane to construct an epithelial-like cell layer with tight junctions, $\beta$-glucan was added to the apical (luminal) 
side of the cells. The amount of $\beta$-glucan transported to the basement membrane side (lamina propria side) was measured over time. When $1 \mathrm{mg}$ of Curdlan solution was added to the apical side of the Caco-2 cells, approximately $500 \mathrm{ng}(250 \mathrm{ng} / \mathrm{ml})$ of Curdlan had been transported to the basement membrane side five hours later (Figure 6). This amount corresponded to $1 / 2000$ th of the $\beta$-glucan added to the apical side, and this amount increased over time. The fact that human intestinal epithelial-like cells transport $\beta$-glucan by polarized transport suggested that the human intestinal tract may also take up $\beta$-glucans into the body. To investigate whether $\beta$-glucan is taken up into human intestinal epithelial-like cells as in the small intestinal tissue of mice, we performed fluorescence immunostaining using soluble Dectin- 1 as a probe and observed that $\beta$-glucans were similarly taken up intracellularly in Caco-2 cells. The possible mechanisms for cellular uptake of extracellular macromolecules are receptor-dependent and receptor-independent endocytosis. To determine the mechanism by which $\beta$-glucans are taken up into Caco- 2 cells, a cytophysiological experiment using three pharmacological agents (Cytochalasin D, Dynasore, Genistein) was conducted. The results showed that intracellular uptake of $\beta$-glucan was notably inhibited only when Cytochalasin D, an actin polymerization inhibitor, was used. Cytochalasin D is known to primarily inhibit processes such as phagocytosis and macropinocytosis. Thus, intestinal epithelial cells may be detecting the $\beta$-glucans through an unknown mechanism and actively taking up and transporting them to the basement membrane side.

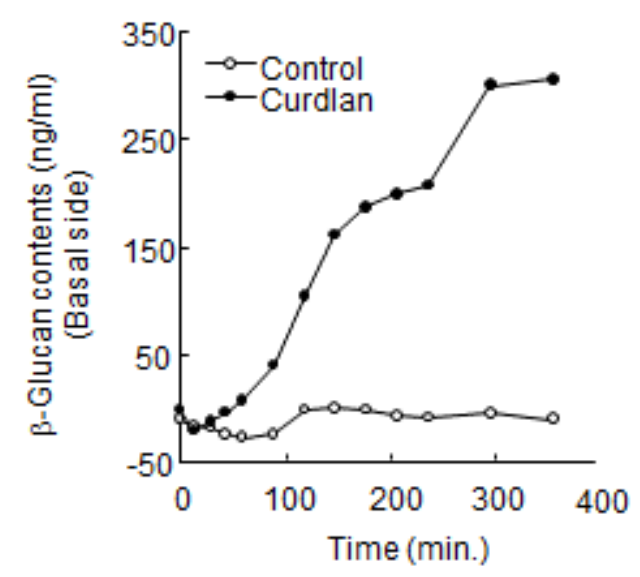

Figure 6 Apical to basolateral transport of $\beta$-glucan in human intestinal epithelial-like Caco-2 cells. Transport of $\beta$-glucan from apical to basolateral side by Caco- 2 cells cultured for 21 days on porous membranes was quantified. One $\mathrm{mg} / \mathrm{mL}$ of curdlan was added to the apical side of Caco- 2 cells and the basolateral solution was collected over time. The $\beta$-glucan transported to the basolateral side was quantified by sandwich ELISA using recombinant mouse Dectin-1.

\subsection{Effect of $\beta$-glucans that are taken up into the body on the immune system}

It was described above that trace amounts of $\beta$-glucans are taken up into the body from the digestive tract, but these are foreign substances to the body, and as mentioned in the second section, they may have various effects on the biological immune system. The digestive tract is one of the most accessible entry points for pathogens such as bacteria and viruses, and thus, there is a well-developed immune organ known as the Gut Associated Lymphoid Tissue (GALT). Furthermore, many immune system cells such as lymphocytes and antigen-presenting cells are present in the lamina propria, and a response towards $\beta$-glucans taken up into the body may be triggered. Therefore, we investigated the effect of $\beta$-glucans transported to the basement membrane side of the Caco- 2 cells on the immune system. Basolateral conditioned medium of Caco- 2 cells cultured with $\beta$-glucan added on the apical side was prepared and added to THP-1 cell medium that was induced to differentiate into macrophages with phorbol ester. After three hours of incubation, induction of tumor necrosis factor 
alpha (TNF- $\alpha$ ) and transforming growth factor beta (TGF- $\beta$ ) production was observed when the induced cytokine expression by THP-1 cells was analyzed using RT-PCR (Figure 7). Since this ability to induce cytokine expression is not deactivated even when heated, it is very likely that it was an immune-activating effect by permeated $\beta$-glucans and not by proteinaceous factors secreted from Caco-2 cells. No such activation was observed when THP-1 cells were directly stimulated by the same amount of $\beta$-glucan as the permeated $\beta$-glucan, indicating that perhaps there is some modification to $\beta$-glucans by intestinal epithelial cells. Alternatively, it is possible that macrophages are being costimulated with heat-resistant humoral factors from Caco-2. Further analysis is required in the future. It has also been reported that immune system cells present in the intestinal lamina propria have suppressed inflammatory responses against PAMPs [26]. Thus, whether the response induced in THP-1 cells actually occurs in the intestinal tract has remained to be established.

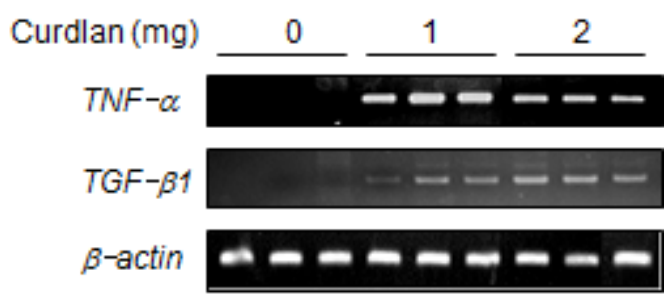

Figure 7 Activation of macrophages by the basolateral solution of Caco-2 cells with $\beta$-glucan added to the apical side. One $\mathrm{mg} / \mathrm{mL}$ of curdlan was added to the apical side of monolayer-cultured Caco-2 cells, and the basolateral solution was collected 3 hours later. For the analysis of macrophage activation, THP-1 cells, a human monocyte cell line, were used. After stimulating THP-1 cells with the basolateral solution for 3 hours, RNA was prepared and RT-PCR was performed.

\section{Conclusion}

This review introduced the intestinal absorption of $\beta$-glucan, an insoluble dietary fiber that has various effects on the immune system. Since the 1980s, studies have reported the various effects of $\beta$ glucans on the immune system, and reports have also been made on receptors, slowly clarifying their recognition system and action mechanisms. However, since most of these studies were focused on treatments of infectious diseases and tumors, much remains unknown about the effects of $\beta$-glucans ingested in food as dietary fiber and their mechanisms of action.

At the present time, the physiological significance of small intestinal epithelial cells absorbing nonnutrient $\beta$-glucans into the body is unknown. However, the studies mentioned above have elucidated that $\beta$-glucans that can bind to receptors and activate immune cells are transported to the basement membrane side. It is also known that immune system cells present in the intestinal tract are activated when microbial components such as adenosine triphosphate (ATP) released by intestinal bacteria, lipopolysaccharides (LPS), which are cell wall components of Gram-negative bacteria, and cytosinephosphate-guanine (CpG) motifs in bacterial deoxyribonucleic acid (DNA) are taken up in the body and maintain homeostasis of the intestinal immune system [27]. In a similar manner, $\beta$-glucans may also be contributing to maintaining intestinal immune homeostasis.

Author Contributions: Conceptualization, S.H. and Tsukasa.M.; methodology, S.H.; formal analysis,S.H. and N.N.; investigation, S.H.; data curation, S.H.; writing - original draft preparation, S.H.; writing- 
review and editing, Tsukasa M.,Tatsuya M, N,N.; funding acquisition, S.H. and Tsukasa M. All authors have read and agreed to the published version of the manuscript.

Funding: This research was funded by by JSPS KAKENHI Grant Number JP26660109 and JP 23780131.

Conflicts of Interest: The authors declare no conflict of interest.References

1. McIntosh M, Stone BA, Stanisich VA: Curdlan and other bacterial (1-->3)-beta-D-glucans. Appl Microbiol Biotechnol 2005;68(2):163-73.

2. Munz C, Steinman RM, Fujii S: Dendritic cell maturation by innate lymphocytes: coordinated stimulation of innate and adaptive immunity. J Exp Med 2005;202(2):203-7.

3. Sasaki T, Takasuka N: Further study of the structure of lentinan, an anti-tumor polysaccharide from Lentinus edodes. Carbohydr Res 1976;47(1):99-104.

4. Tabata K, Ito W, Kojima $\mathrm{T}$ et al.: Ultrasonic degradation of schizophyllan, an antitumor polysaccharide produced by Schizophyllum commune Fries. Carbohydr Res 1981;89(1):121-35.

5. Garcia-Lora A, Martinez M, Pedrinaci S et al.: Different regulation of PKC isoenzymes and MAPK by PSK and IL-2 in the proliferative and cytotoxic activities of the NKL human natural killer cell line. Cancer Immunol Immunother 2003;52(1):59-64.

6. Sato M, Sano H, Iwaki D et al.: Direct binding of Toll-like receptor 2 to zymosan, and zymosaninduced NF-kappa B activation and TNF-alpha secretion are down-regulated by lung collectin surfactant protein A. J Immunol 2003;171(1):417-25.

7. Kurashige S, Akuzawa Y, Endo F: Effects of Lentinus edodes, Grifola frondosa and Pleurotus ostreatus administration on cancer outbreak, and activities of macrophages and lymphocytes in mice treated with a carcinogen, N-butyl-N-butanolnitrosoamine. Immunopharmacol Immunotoxicol 1997;19(2):175-83.

8. Schmid F, Stone BA, McDougall BM et al.: Structure of epiglucan, a highly side-chain/branched (1 --> 3;1 --> 6)-beta-glucan from the micro fungus Epicoccum nigrum Ehrenb. ex Schlecht. Carbohydr Res 2001;331(2):163-71.

9. Ladanyi A, Timar J, Lapis K: Effect of lentinan on macrophage cytotoxicity against metastatic tumor cells. Cancer Immunol Immunother 1993;36(2):123-6.

10. Hetland G, Ohno N, Aaberge IS et al.: Protective effect of beta-glucan against systemic Streptococcus pneumoniae infection in mice. FEMS Immunol Med Microbiol 2000;27(2):111-6. 
11. Suzuki I, Tanaka H, Kinoshita A et al.: Effect of orally administered beta-glucan on macrophage function in mice. Int J Immunopharmacol 1990;12(6):675-84.

12. Hong F, Yan J, Baran JT et al.: Mechanism by which orally administered beta-1,3-glucans enhance the tumoricidal activity of antitumor monoclonal antibodies in murine tumor models. J Immunol 2004;173(2):797-806.

13. LeibundGut-Landmann S, Gross O, Robinson MJ et al.: Syk- and CARD9-dependent coupling of innate immunity to the induction of T helper cells that produce interleukin 17. Nat Immunol $2007 ; 8(6): 630-8$.

14. Dillon S, Agrawal S, Banerjee K et al.: Yeast zymosan, a stimulus for TLR2 and dectin-1, induces regulatory antigen-presenting cells and immunological tolerance. J Clin Invest 2006;116(4):91628.

15. Lehne G, Haneberg B, Gaustad P et al.: Oral administration of a new soluble branched beta-1,3D-glucan is well tolerated and can lead to increased salivary concentrations of immunoglobulin A in healthy volunteers. Clin Exp Immunol 2006;143(1):65-9.

16. Masuzawa S, Yoshida M, Ishibashi K et al:: Solubilized candida cell Wall @-glucan, CSBG, is an epitope of natural human antibody. Drug Dev. Res. 2003;58(2):179-189.

17. Ikeda Y, Adachi Y, Ishii T et al.: Dissociation of Toll-like receptor 2-mediated innate immune response to Zymosan by organic solvent-treatment without loss of Dectin-1 reactivity. Biol Pharm Bull 2008;31(1):13-8.

18. Ross GD, Vetvicka V: CR3 (CD11b, CD18): a phagocyte and NK cell membrane receptor with multiple ligand specificities and functions. Clin Exp Immunol 1993;92(2):181-4.

19. Yan J, Vetvicka V, Xia Y et al.: Beta-glucan, a "specific" biologic response modifier that uses antibodies to target tumors for cytotoxic recognition by leukocyte complement receptor type 3 (CD11b/CD18). J Immunol 1999;163(6):3045-52.

20. Brown GD: Dectin-1: a signalling non-TLR pattern-recognition receptor. Nat Rev Immunol 2006;6(1):33-43.

21. Gantner BN, Simmons RM, Canavera SJ et al.: Collaborative induction of inflammatory responses by dectin-1 and Toll-like receptor 2. J Exp Med 2003;197(9):1107-17. 
22. Brown GD, Gordon S: Immune recognition. A new receptor for beta-glucans. Nature 2001;413:3637.

23. Ujita M, Nagayama H, Kanie $S$ et al.: Carbohydrate binding specificity of recombinant human macrophage beta-glucan receptor dectin-1. Biosci Biotechnol Biochem 2009;73(1):237-40.

24. Matsuda T, Matsubara T, Hino S: Immunogenic and allergenic potentials of natural and recombinant innocuous proteins. J Biosci Bioeng 2006;101(3):203-11.

25. Rice PJ, Adams EL, Ozment-Skelton T et al.: Oral delivery and gastrointestinal absorption of soluble glucans stimulate increased resistance to infectious challenge. J Pharmacol Exp Ther 2005;314(3):1079-86.

26. Smythies LE, Sellers M, Clements RH et al: Human intestinal macrophages display profound inflammatory anergy despite avid phagocytic and bacteriocidal activity. J Clin Invest 2005;115(1):66-75.

27. Abt MC, Artis D: The intestinal microbiota in health and disease: the influence of microbial products on immune cell homeostasis. Curr Opin Gastroenterol 2009;25(6):496-502. 